\title{
APORTACIONES DE LOS REMONTAJES ÓSEOS EN LOS ESTUDIOS ZOOARQUEOLÓGICOS, TAFONÓMICOS Y DE ARQUEOLOGÍA ESPACIAL
}

\author{
María Cristina Fernández-Laso ${ }^{1}$
}

\section{Resumen:}

Los remontajes óseos pueden aportarnos información relevante en los estudios zooarqueológicos, tafonómicos y de arqueología espacial. En zooarqueología nos permiten, entre otros, la reconstrucción de los huesos fracturados, mejorar los porcentajes de identificación anatómica y taxónomica de los restos óseos, y el cálculo del número de elementos identificados por especie (NISP), el número mínimo de elementos (NME) y el número mínimo de individuos (NMI); también nos permiten evaluar la integridad de un conjunto óseo, e inferir aspectos del procesamiento de animales por parte de los humanos, carnívoros u otros agentes tafonómicos. En los estudios tafonómicos nos aportan por ejemplo información sobre los procesos de formación de los yacimientos arqueológicos, nos permiten resolver problemas estratigráficos, reconocer desplazamientos horizontales/ verticales de los huesos, o identificar procesos postdeposicionales. $\mathrm{Y}$ en la arqueología espacial, en Paleolítico, pueden ayudarnos en la reconstrucción de los patrones de movilidad de los homínidos, por ejemplo identificar áreas de actividad, procesado de animales, o su relación con el espacio ocupado. Este artículo se centra en los trabajos sobre remontajes óseos realizados en yacimientos del Paleolítico, y en sus aportaciones en los estudios zooarqueológicos, tafonómicos y de arqueología espacial.

\section{Palabras clave:}

remontajes óseos, zooarqueología, tafonomía, arqueología espacial, Paleolítico.

\section{Abstract:}

Bone refits can provide relevant information on zooarchaeological, taphonomical and spatial archaeology studies. In zooarchaeology they allow us, among other things, to reconstruct fractured bones, increase the percentages of anatomical and taxonomical identification of skeletal remains, and determine the number of elements identified by species (NISP), the minimum number of elements (MNE) and the minimum number of individuals (MNI); they also enable us to assess the integrity of a bone assemblage, and infer aspects of the processing of animals by humans, carnivores or other taphonomical agents. In taphonomical studies they provide us, for instance, with information on the formation processes of archaeological sites, allow us to solve stratigraphical problems, recognize horizontal/vertical

1 Universidad Internacional de La Rioja (UNIR). C/Almanza 101, 28040 Madrid cristina.fernandezl@unir.net 
displacements of the bones, or identify postdepositional processes. As for spatial archaeology, in Paleolithic sites, they can help in the reconstruction of mobility patterns of hominids, identifying, for example, areas of activity, animal processing or relationship with the occupied space. This paper focuses on the publications on bone refits in Paleolithic sites, and their contributions to zooarchaeological, taphonomical and spatial archaeology studies.

\section{Key words:}

Bone refits, zooarchaeology, taphonomy, spatial archaeology, Paleolithic.

\section{Introducción}

Los remontajes en arqueología se refieren a la reconstrucción de fragmentos fósiles fracturados independientemente de su naturaleza (huesos, lítica, cerámica, vidrio, etc.). En tecnología lítica los remontajes definen la reconstrucción de secuencias temporales de una cadena operativa parcial o completa, por tanto hacen referencia a la recomposición de la explotación de un núcleo; mientras que en zooarqueología se definen por la reconstrucción anatómica de los elementos y/o del esqueleto de un animal (Cziesla et al., 1990; Todd y Stanford 1992). Los remontajes es una técnica utilizada en arqueología desde las últimas décadas del siglo XIX (Spurrell 1880). Tiene su origen en el análisis de la industria lítica y su uso comienza a estandarizarse a partir de los años noventa del siglo XX, tanto en Europa como en Norteamérica (Cziesla et al., 1990; Schurmans 2007). Y a partir de este momento esta técnica también se extiende a otro tipo de materiales arqueológicos como los huesos (Hofman y Enloe 1992). Los primeros trabajos sobre remontajes en Paleolítico se Ilevan a cabo en los yacimientos del Paleolítico superior, siendo más habituales en lítica que en huesos, y en los yacimientos al aire libre que en las cuevas o abrigos (e.g. Roebroeks 1988; Locht 2001; Conard et al., 1998; Vaquero et al., 2001). La mayoría de estos primeros trabajos acuden a los remontajes como un método complementario para reforzar sus interpretaciones arqueológicas. Es decir que rara vez están centrados exclusivamente en los remontajes como método para interpretar por ejemplo los comportamientos humanos en el pasado, o para resolver problemas estratigráficos, o procesos postdeposicionales, etc.

En general, en la actualidad las publicaciones sobre remontajes son escasas y esto se debe, entre otros, a la inversión de tiempo y coste económico que requieren (Larson y Ingbar 1992; Cooper y Qiu 2006) y, como veremos, a las propias limitaciones que presentan los huesos en numerosos conjuntos arqueológicos. No obstante, en la última década se ha observado un relativo aumento de estos trabajos, aunque más en tecnología lítica que en zooarqueología. Esto es debido probablemente a: a) una mayor concienciación de los complejos procesos de 
formación de los yacimientos, b) el interés por los procesos cognitivos de las distintas especiales de homínidos en la articulación del espacio ocupado, c) y por la necesidad de desarrollar nuevos métodos y técnicas para leer e interpretar el registro arqueológico. Además, la incorporación de las tecnologías de la información y la comunicación (TIC) en la práctica arqueológica, con la inclusión de avanzados y sofisticados software y escáneres 3D, ha facilitado también la proliferación de este tipo de trabajos, sin embargo estos siguen siendo aún escasos.

Este artículo se centra en una breve revisión de los estudios sobre remontajes óseos realizados hasta hoy en día, y en su contribución en los estudios zooarqueológicos, tafonómicos y de arqueología espacial. Los remontajes óseos como afirmaba Enloe (1995) son una técnica multidimensional con una gran ventaja de aplicaciones y potenciales. No obstante, no parece que desde esta afirmación, hace ya más de dos décadas, hayan proliferado de forma notable su realización y aplicación en el análisis de los yacimientos arqueológicos.

\section{Los remontajes óseos}

En un yacimiento arqueológico todo fragmento óseo identificado es potencialmente susceptible de unirse o remontar con otro fragmento óseo (Hofman 1992a). Los remontajes son un puzle complejo de restos óseos o partes esqueléticas, en el que suelen estar presentes distintos puzles al mismo tiempo; es decir más de un esqueleto de un animal, y en el que además este esqueleto o esqueletos pueden estar completos o bien faltarnos numerosas piezas (Lyman 1994). Así, la complejidad de los remontajes óseos se incrementa conforme aumenta el número de esqueletos de uno o varios taxones, y también conforme aumenta la intervención de los agentes y procesos de alteración tafonómica en la formación de la acumulación ósea. Esto es precisamente una de las principales causas que determinan la ausencia de este tipo de trabajos en los yacimientos arqueológicos.

En los remontajes de huesos se distinguen cuatro métodos: 1) mecánicos, 2) articulaciones, 3) las parejas bilaterales, y 4) las articulaciones del mismo miembro.

1) Los remontajes mecánicos (Todd 1987; Lyman 1994) consisten en la acción mecánica de conectar dos o más fragmentos pertenecientes a un mismo elemento o porción esquelética; por tanto esto comporta la conexión entre dos planos de fractura, o bien la conexión de un positivo-negativo en el caso de desprendimientos medulares o corticales (conos de percusión, lascas) de un hueso. Este tipo de remontajes son los más "fáciles" de realizar dada la naturaleza de los materiales y también es el método más abundante en la literatura arqueológica (e.g. Hofman y Enloe 1992; Hofman 1986; 1992; Villa 1982; 1991; Henshaw 1999; Bravo 2001; 
Marín Arroyo 2004; Morin et al., 2005; Fernández-Laso 2010; Rosell et al., 2012; Gabucio 2014). Generalmente se utilizan para facilitar la identificación de los fragmentos indeterminados anatómica y taxonómicamente (e.g. Marean y Kim, 1998). Los primeros trabajos de este tipo proceden del yacimiento de Pincevent (Francia) que reconstruyen los huesos largos con el fin de estudiar los patrones de dispersión de los elementos, y de definir los límites de las áreas de ocupación (Leroi-Gourhan y Brézillon 1966; 1972).

Ahora bien, los remontajes no consisten exclusivamente en la reconstrucción de los elementos. La reconstrucción de un elemento no constituye el proceso final del remontaje, sino más bien es el principio (Hofman 1986). Se trata de un método que por sí mismo aporta información, pero que aumenta su potencial con un trabajo multidisciplinar para inferir, entre otros, la naturaleza de las relaciones de los remontajes. Es decir la distribución espacial de los elementos y su análisis e interpretación espacial y temporal. En otras palabras, la reconstrucción de un elemento nos aporta información por sí mismo, desde un punto de vista anatómico y taxónomico, pero continua siendo un ítem estático (Binford 1988), mientras que la combinación con la tafonomía y la arqueología espacial lo convierte en un ítem dinámico, que contiene información en el espacio y en el tiempo. Los remontajes permiten reconstruir los actos del individuo o de los grupos en el pasado, en relación con una actividad o actividades de subsistencia, desde la fracturación de un fragmento al descuartizamiento de distintos taxones en secuencias de actividades temporales (continuadas o interrumpidas). Por tanto, permiten observar la planificación y consecución de actividades (Hallos 2005; Fernández-Laso, 2010).

En los remontajes mecánicos se diferencian, en función del origen de las roturas de los huesos, tres tipos (Todd y Stanford 1992): a) remontajes entre las fracturas en fresco, son aquellos que presentan más dificultades a la hora de realizar reconstrucciones, ya que los huesos hasta que se fosilizan están cambiando sus bordes, al menos durante algunos años (Morín et al., 2005). Los humanos y los carnívoros son los agentes que producen más fracturas y modificaciones en los huesos. Los humanos por ejemplo en la acción de precalentar los huesos en el fuego para facilitar su fracturación, o en la elaboración de herramientas u objetos decorativos modifican los bordes de fractura y, por consiguiente, dificultan o imposibilitan las tareas de remontar los huesos. Los carnívoros con el mordisqueo de los bordes de fractura de los huesos, con la acción de los ácidos salivares o con la digestión, etc., pueden también hacer difícil la reconstrucción de los elementos. b) Remontajes entre las fracturas en seco, estos pueden aportarnos información sobre los movimientos o desplazamientos que se han producido en los restos con posterioridad a la actividad de los humanos y/o demás agentes tafonómicos. Los remontajes con este tipo fracturas permitió a Todd y Stanford (1992) reconocer que los desplazamientos de los huesos eran postdeposicionales en el yacimiento paleoindio Johns-Miller (Estados Unidos). c) Y finalmente las fracturas 
mixtas, que son aquellos fragmentos óseos que por sus características no pueden incluirse en ninguno de los dos tipos anteriores, ya que combinan rasgos de ambos. Estos generalmente se incluyen en el primer tipo.

2) Los remontajes anatómicos consisten en unir diferentes partes esqueléticas pertenecientes a un mismo individuo; es decir identificar y reagrupar elementos anatómicos contiguos de un mismo lado o del lado contrario de un animal. Se distinguen dos tipos (Todd y Frison 1992; Lyman 1994): a) las parejas bilaterales o elementos anatómicos de ambos lados de un mismo individuo, por ejemplo el húmero derecho e izquierdo de un mismo animal; b) y las articulaciones del mismo miembro (Todd 1987), que consiste en remontar por ejemplo un fémur distal con una tibia proximal del mismo lado de un individuo. La dificultad de este tipo de remontajes aumenta conforme se incrementa el número de individuos en el registro óseo. Ambos tipos precisan conocer la talla, la edad y el sexo de los animales, así como los caracteres métricos y morfológicos de cada elemento esquelético (Todd y Stanford 1992). La realización de estos remontajes requiere (Todd y Frison 1992): a) el registro exhaustivo de las medidas de todos los elementos anatómicos, b) la edad y el sexo de los individuos, c) comparar las medidas de las parejas de elementos y seleccionarlas documentando los rangos de variación y de error en cada elemento del individuo, d) evaluar visualmente el potencial de las parejas bilaterales, observar la distribución de los elementos intentando juzgar la procedencia espacial de las parejas bilaterales de un mismo individuo, f) y calcular las distancias entre las parejas bilaterales.

Esta clase de remontajes es muy habitual entre los elementos del esqueleto craneal, en concreto con la dentición, ya que se pueden reconocer "fácilmente". Es relativamente sencillo emparejar las hileras de los dientes de la derecha y la izquierda gracias a la forma de las cúspides, de las medidas, la alternancia de las bandas de la dentina, del esmalte, y el desgaste global de los dientes. Leroi-Gourhan (1972) en Pincevent (Francia) realiza series dentarias de la parte superior e inferior del reno en la sección 36, que posteriormente será ampliado por Enloe (1991). Sin embargo, este tipo de remontajes para la reconstrucción del esqueleto postcraneal es más compleja. Diferentes investigadores (Enloe 1991; Todd y Frison 1992; Lyman 2006) han llevado a cabo muestras de control de esqueletos de animales modernos como marco referencial, que permita realizar comparativas analíticas con las acumulaciones óseas arqueológicas, pero este tipo de reconstrucciones presenta grandes dificultades y limitaciones. Lyman (2006) señala errores tipo que pueden producirse como: ¿cuántas parejas simétricas bilaterales que hay en el conjunto óseo no son identificadas? ¿Cuántas son identificadas de forma errónea? es decir ¿cuántos elementos emparejados corresponden a distintos animales? Otra problemática principal para la realización de estos remontajes es la imposibilidad de llevarse a cabo en algunas acumulaciones óseas, porque las medidas que se deben tomar en los elementos no pueden ser anotadas dada 
la excesiva fragmentación y rotura que los huesos presentan. Es habitual en los yacimientos arqueológicos, especialmente en el Paleolítico, que los conjuntos óseos muestren altos grados de fragmentación debido a los intensos procesos de carnicería y preparación llevadas a cabo por los homínidos. A lo que se suma una posible intervención de los carnívoros, o acumulaciones generadas por éstos. Por ello en los trabajos sobre remontajes óseos predomina la combinación de sólo dos tipos: los mecánicos y los de articulaciones (e.g. Jonhson 1982; 1987; Enloe y David 1989; Enloe 1991; Todd y Frison 1992; Rapson y Todd 1992; Marean y Kim 1998; Conard et al., 1998; Bravo 2001; Waguespack et al., 2002; Morin et al., 2005; Marín 2004; Fernández-Laso 2010; Rosell et al., 2012; Gabucio 2014).

\section{Aportaciones de los remontajes óseos}

En arqueología los remontajes óseos se han llevado a cabo principalmente desde tres ámbitos (Tabla 1): 1) la tafonomía, como herramienta para entender los procesos de formación de un yacimiento, para resolver cuestiones relacionadas con los movimientos verticales de los huesos y para evaluar procesos postdeposicionales (e.g. Hofman, 1981; Bunn et al., 1980; Villa 1982; Lyman 1989; Morin et al., 2005).

2) La arqueología espacial, para identificar e inferir patrones de comportamiento humano, como identificar áreas de actividad y valorar aspectos de la organización espacial, social e intentar distinguir eventos de ocupación (e.g. Leroi-Gourhan y Brézillon 1972).

3) Y la paleoeconomía, para reconocer la composición de las acumulaciones óseas, reconstruir las estrategias de adquisición de las presas (caza/carroñeo), el tipo de procesamiento y su interacción con otros predadores (Marean y Kim, 1998), valorar las estrategias económicas desarrolladas por los humanos en sus ocupaciones, el tipo de procesamiento y de reparto de los recursos cárnicos (e.g. Larson e Ingbar 1992; Johnson 1987; Parkington et al., 1992; Waguespack et al., 2002).

En un yacimiento arqueológico los remontajes óseos han sido tradicionalmente utilizados como método para esclarecer posibles distorsiones espacio-temporales, es decir para intentar hacer comprensible los palimpsestos (e.g. Villa 1982). Sin embargo, los huesos no sólo se desplazan tras la desocupación humana por la acción de procesos postdeposicionales. Los huesos no son ítems estáticos que yacen en el substrato de forma inamovible durante las tareas de procesamiento, preparación, cocinado y consumo antrópico. Durante estas tareas puede llevarse a cabo el desplazamiento de elementos por causas como: a) el procesado de un animal por más de un individuo, es decir la división en cuartos y su reparto en distintas áreas del espacio ocupado, b) por la compartición de la comida, y/o por la limpieza del hábitat. Así Todd y Stanford (1992) señalan que los 
huesos largos fracturados por los humanos para la obtención de la médula se encuentran muy próximos entre sí; y que generalmente las distancias mayores de los elementos pueden responder a tareas de limpieza del lugar o a la acción de otros agentes, como pueden ser los carnívoros. Ciertamente, las actividades de descuartizamiento de los esqueletos de los animales requiere de un espacio que no necesariamente tiene que estar asociado con los hogares (Binford, 1983), por lo que tras dicha actividad es lógico pensar en el desplazamiento de los elementos para su reparto, cocinado, consumo, abandono o eliminación. En consecuencia, la dispersión de los huesos del esqueleto de un animal puede ser el resultado de la preparación y de su consumo, y no únicamente de los procesos de formación postdeposicionales (Schiffer, 1983).

El trabajo de Rapson y Todd (1992) es un buen ejemplo en este sentido. Estos autores realizan remontajes mecánicos y de articulaciones de los elementos de la oveja y el bisonte para examinar la contemporaneidad y la estructura del yacimiento de Bugas-Holding (Estados Unidos). La observación de las fracturas y su dispersión les lleva a concluir desplazamientos de los elementos desde su fractura hasta el fuego más próximo, y de ahí hasta la rotura y abandono en otro fuego. Éstos observan trayectorias distintas en función de las especies, lo que les permite evaluar patrones diferentes de comportamiento en función de los taxones.

Previamente, Leroi-Gourhan y Brézillon (1972), a través de las reconstrucciones de los huesos largos de Pincevent (Francia), reconstruyen los patrones de dispersión y definen los límites de las áreas de ocupación. Estos autores atribuyen las distancias entre los elementos a actividades de mantenimiento y de limpieza, y sugieren un amplio abanico de actividades y un patrón de ocupación complejo. Posteriormente, este estudio es ampliado por Enloe y David (1989; 1992) quienes realizan remontajes mecánicos y anatómicos para examinar los patrones de distribución de los alimentos. Observan ocupaciones contemporáneas, animales compartidos entre tres áreas que se corresponden con grupos distintos. Enloe (1991) aumenta el área de estudio para incluir los materiales procedentes de otros 6 hogares, aumentando la distancia de distribución de los alimentos hasta alcanzar los 63 metros. Este autor comprueba que el número y la intensidad de las relaciones son inversamente proporcionales a la distancia entre los hogares. Por tanto, demuestra que la proximidad favorece una mayor interacción económica entre los grupos humanos (Gargget y Hayden, 1991), y que la repartición de los alimentos difiere de acuerdo con la parte del esqueleto del animal. Así comprueba que las extremidades superiores (tibia y fémur) ricas en carne tienen mayor circulación que la parte distal de las extremidades (metapodios) ricas en médula. Estas últimas no son distribuidas entre las áreas de actividad (Enloe y David 1989; 1992; Todd y Frison 1992). Johnson (1987) en el yacimiento de Lubbock Lake (Estados Unidos) utiliza los remontajes mecánicos y anatómicos para identificar patrones y áreas de descuartizamiento de bisontes. En esta misma línea se 
desarrolla el trabajo de Parkington et al., (1992) en el yacimiento de Dunefield Midden Campsite (África) con los remontajes de elementos de antílopes. Otro interesante trabajo sobre la distribución y reparto de comida es el de Waguespack et al., (2002) en el yacimiento de Palangana (Alaska) con los remontajes realizados con los restos de caribou. Bravo (2001) en el nivel Ja del yacimiento Abric Romaní (Capellades, Barcelona) aporta con los remontajes importante información sobre los procesos de fracturación y de dispersión de los restos por las actividades de los neandertales, y por los procesos postdespocionales. Posteriormente se realizan los remontajes de los niveles arqueólogicos posteriores: K, L y M (Fernández-Laso, 2010). Los remontajes mecánicos y anatómicos nos permiten identificar también actividades de procesado de animales y relacionar distintas áreas de actividad de los neandertales (Figura 1). A este trabajo, le siguen en esa misma línea las publicaciones de Rosell et al., (2012) para el nivel H y Ja, y de Gabucio (2014) para el nivel O (Tabla 2).

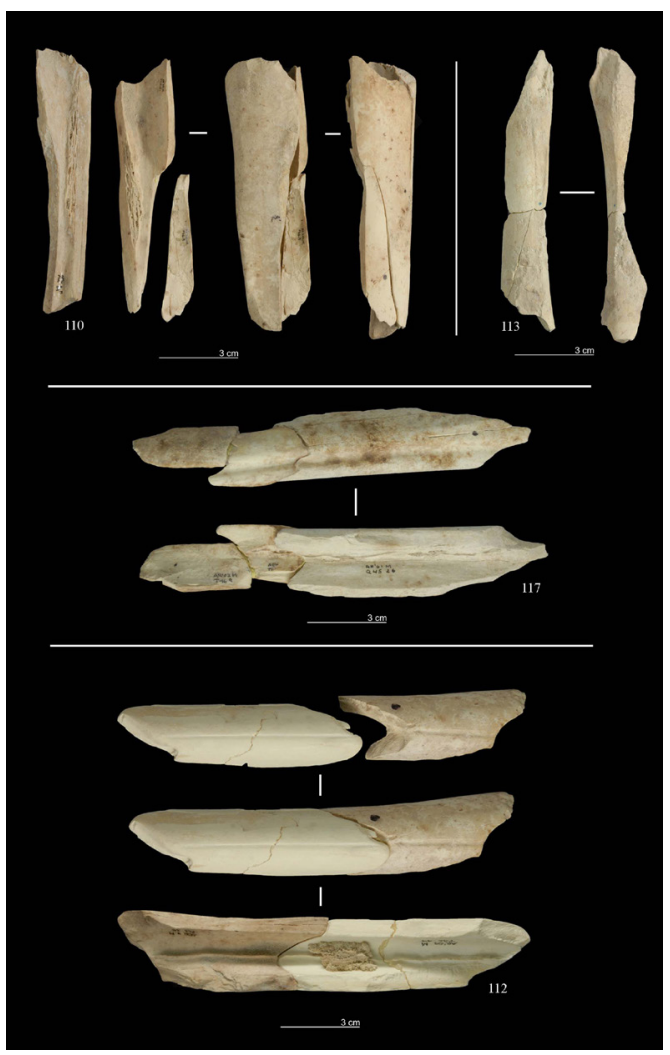

Fig. 1. Ejemplos de remontajes óseos de tipo mecánico identificados en el nivel $\mathrm{M}$ del yacimiento Abric Romaní (Barcelona, España). 110: remontaje de tres fragmentos de radio de cérvido; 112: remontaje de tres restos de metacarpo de cérvido; 113: remontaje de dos fragmentos de fémur de cérvido; 117: remontaje de tres restos metatarso de cérvido. Foto: Gerard Campeny (IPHES). 
En resumen, los remontajes óseos dispersados de las carcasas de los animales en un campamento pueden informarnos sobre las secuencias y los procedimientos del procesado, y sobre la contemporaneidad de las actividades llevadas a cabo (Audouze y Enloe, 1997; Fernández-Laso 2010).

Ciertamente, es indiscutible que dos o más fragmentos óseos remontados son contemporáneos entre sí, ya que éstos se separan simultáneamente, en el mismo momento. Sin embargo, los eventos tafonómicos subsiguientes que tienen lugar en el fragmento/s hasta que es recuperado por el arqueólogo no tienen obviamente porque ser contemporáneos (Hofman 1981; Larson e Ingbar, 1992; Lyman 2008). En este sentido, es necesario evaluar todos los procesos y agentes que intervienen en la formación de un conjunto óseo. Es esto precisamente una de las principales diferencias con los remontajes líticos, es decir la ausencia de reciclaje en los elementos óseos detectada en la industria lítica (Vaquero 2011; Vaquero et al., 2015); y es lo que hace enormemente interesante la realización conjunta de remontajes óseos y líticos en los yacimientos arqueológicos, pues nos aportan diferente información como mínimo temporal.

\begin{tabular}{|c|c|c|}
\hline Zooarqueología & Tafonomía & Arqueología Espacial \\
\hline 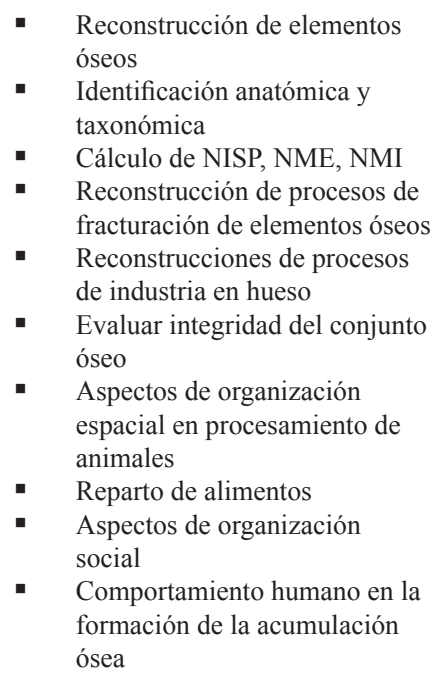 & $\begin{array}{l}\text { Reconstrucción de Procesos } \\
\text { de formación de los } \\
\text { yacimientos } \\
\text { Reconstrucciones espacio- } \\
\text { temporales } \\
\text { - Sincronía/ } \\
\text { Contemporaneidad } \\
\text { - Diacronía/reconocimiento } \\
\text { de movimientos } \\
\text { verticales intraestratos, } \\
\text { transestratigráficos } \\
\text { Resolución de problemas } \\
\text { de integridad estratigráfica } \\
\text { - Identificación de procesos } \\
\text { naturales } \\
\text { - Identificación de procesos } \\
\text { postdeposicionales } \\
\text { - Evolución de la formación } \\
\text { del registro arqueológico } \\
\text { - Integridad contextual }\end{array}$ & $\begin{array}{l}\text { - } \begin{array}{l}\text { Reconstrucción detallada áreas } \\
\text { de actividad }\end{array} \\
\text { Relaciones temporales entre las } \\
\text { acumulaciones o las unidades } \\
\text { espaciales } \\
\text { - } \begin{array}{l}\text { Reconstrucción de patrones } \\
\text { de movilidad entre área de }\end{array} \\
\text { actividad intrasite } \\
\text { - Relación entre la espacio y } \\
\text { registro óseo } \\
\text { Reconstrucción de patrones de } \\
\text { comportamiento } \\
\text { Articulación del espacio } \\
\text { relación con estructuras }\end{array}$ \\
\hline
\end{tabular}

Tabla 1. Aportaciones de los remontajes óseos en los estudios de zooarqueología, tafonomía y arqueología espacial. 
Además de la acción de los homínidos otros agentes tafonómicos que pueden producir desplazamientos en los huesos son: la desarticulación natural (Andrews y Cook, 1985), la acción de carnívoros (e.g. Camaros et al., 2013), los roedores (Brain 1981), las corrientes hídricas, el viento, el hielo, la gravedad, el pisoteo o trampling (e.g. Voorhies 1969; Stevenson 1991; Lyman 1989; 1994; Blasco et al., 2008). Los remontajes óseos han sido utilizados para evaluar posibles procesos postdeposicionales (Bunn et al., 1980; Villa 1982; et al., 1986; Lyman 1989; Hofman 1992b; Todd y Stanford 1992; Morin et al., 2005). Según Villa (1982) es necesario proceder a los remontajes para ver si los materiales aparentemente in situ corresponden realmente al mismo intervalo de tiempo, previo a realizar agrupaciones de los conjuntos e interpretar actividades. Esta autora considera que muchas interpretaciones de secuencias microestratigráficas necesitan tratarse con precaución y leerse críticamente. En este sentido, apuesta por los remontajes como una herramienta no solo para definir áreas de actividad como en Pincevent (Francia) o Meer II (Bélgica), sino para investigar y entender mejor las limitaciones y complejidad del registro estratigráfico (Tabla 2).

En este sentido, Morin et al., (2005) en el yacimiento de St. Cesaire (Francia) llevan a cabo remontajes mecánicos y anatómicos procedentes de 8 conjuntos, con el fin de intentar identificar distintas ocupaciones que forman parte de un palimpsesto. Estas ocupaciones se corresponden con momentos culturalmente distanciados, Chatelperroniense y Musteriense, pero mezclados al descubrirse en 1979 un esqueleto de neandertal asociado con industrias del primer tipo. Estos autores comprueban con los remontajes que las fracturas postdeposicionales proporcionan información sobre mezcla de elementos en una parte de las ocupaciones. Por el contrario, los remontajes con fracturas en fresco se localizan próximos entre sí, indicando ausencia de movimientos postdeposicionales. Previamente Villa et al., (1986) analizan en el yacimiento de Fontbregoua (Salernes-var) remontajes anatómicos de elementos esqueléticos que tenían una procedencia estratigráfica vertical distinta, identificando grupos de huesos procesados y dispersados al mismo tiempo. Estos investigadores comprueban que los desplazamientos y la distancia entre estos se debió a procesos postdeposicionales. Todd y Stanford (1992) en el cazadero paleoindio JonesMiller en (Estado Unidos) examinan los remontajes de los huesos fracturados en estado seco por exposición a los agentes atmosféricos, e identifican los desplazamientos horizontales que han padecido los huesos tras las actividades de caza y descuartizamiento de los animales por parte de los humanos, es decir los movimientos son postocupacionales. Otro trabajo excepcional que combina remontajes con arqueología espacial y experimentación es el de Bunn et al., (1980) donde identifican en el yacimiento Fxjj50 (Kenia) patrones de distribución horizontal como consecuencia de la actividad de los homínidos y los desplazamientos y las agrupaciones de elementos, tanto horizontales como verticales, por la actuación de otros agentes tafonómicos. 


\begin{tabular}{|c|c|c|c|}
\hline Yacimiento & Cronología & Tipo Remontaje & Publicación \\
\hline Pincevent, Francia & Paleolítico superior & $\begin{array}{l}\text { Mecánico } \\
\text { Anatómico }\end{array}$ & $\begin{array}{l}\text { Leroi-Gourhan y Brézillon } \\
1972 \\
\text { Enloe y David 1989; } 1992\end{array}$ \\
\hline FxJj50, Kenia & Paleolítico inferior & Mecánico & Bunn et al., 1980 \\
\hline Terra Amata, Francia & Paleolítico Inferior & Mecánico & Villa 1982 \\
\hline Koobi Fora, & Paleolítico Inferior & Mecánico & Kroll y Isaac 1984 \\
\hline Fontbrégoua Cave, Francia & Neolítico & Anatómico & Villa et al., 1986 \\
\hline Lubbock Lake & Paleoindio & $\begin{array}{l}\text { Mecánico } \\
\text { Anatómico }\end{array}$ & Johnson 1987 \\
\hline Olduvai Gorge & Paleolítico inferior & Mecánico & Bunn y Kroll 1987 \\
\hline Lubock Lake, EE.UU & Paleoindio & $\begin{array}{l}\text { Mecánico } \\
\text { Anatómico }\end{array}$ & Johnson 1987 \\
\hline Bugas-Holding, EE.UU & Paleoindio & $\begin{array}{l}\text { Mecánico } \\
\text { Anatómico }\end{array}$ & Rapson y Todd 1992 \\
\hline Jones-Miller, EE.UU & Paleoindio & $\begin{array}{l}\text { Mecánico } \\
\text { Anatómico }\end{array}$ & Todd y Stanford 1992 \\
\hline Horner, EE.UU & Paleoindio & $\begin{array}{l}\text { Mecánico } \\
\text { Anatómico } \\
\text { Parejas bilaterales }\end{array}$ & Todd y Frisson 1992 \\
\hline Dunefield Midden Campsite & Paleolítico superior & $\begin{array}{l}\text { Mecánicos } \\
\text { Anatómicos }\end{array}$ & Parkington et al., 1992 \\
\hline Rhineland, Alemania & Paleolítico medio & Mecánicos & Conard et al., 1998 \\
\hline $\begin{array}{l}\text { Champréveyres } \\
\text { Monruz, Suiza }\end{array}$ & Paleolítico superior & Anatómico & Morell, 1994 \\
\hline Abric Romaní, España & Paleolítico medio & $\begin{array}{l}\text { Mecánico } \\
\text { Anatómico }\end{array}$ & $\begin{array}{l}\text { Bravo } 2001 \\
\text { Chacón y Fernández-Laso } \\
2007 \\
\text { Fernández-Laso } 2010 \\
\text { Rosell et al., } 2012 \\
\text { Gabucio } 2014\end{array}$ \\
\hline Palangana, Alaska & Paleolítico superior & $\begin{array}{l}\text { Mecánico } \\
\text { Anatómico }\end{array}$ & Waguespack et al., 2002 \\
\hline Cueva Mirón, España & Paleolítico superior & $\begin{array}{l}\text { Mecánico } \\
\text { Anatómico }\end{array}$ & Marín Arroyo 2004; 2007 \\
\hline Sant Cesaire, Francia & $\begin{array}{l}\text { Paleolítico medio } \\
\text { Paleolítico superior }\end{array}$ & Mecánico & Morin et al., 2005 \\
\hline
\end{tabular}

Tabla 2. Yacimientos arqueológicos con estudios sobre remontajes óseos. 
Kroll y Isaac (1984), por su parte, realizan en Koobi Fora (Kenia) remontajes para evaluar los patrones de dispersión y los movimientos verticales de algunos elementos que, por la acción de procesos postdeposicionales y por la propia orografía de la superficie, ha mezclado momentos ocupacionales distintos. Marín Arroyo (2004) realiza también remontajes en el yacimiento de la Cueva del Mirón (Cantabria) para identificar procesos postdeposicionales posteriores a la ocupación humana. Esto a su vez le permite reconstruir algunas secuencias de fracturación antrópica (Tabla 2).

Los remontajes constituyen, por tanto, una forma de resolver problemas microestratigráficos, de mezcla de ocupaciones breves continuadas; es decir permiten diferenciar estratigráficamente unidades mínimas de ocupación, las cuales pueden corresponder a sencillos episodios o eventos de ocupación superpuestos pero con características estratigráficas similares (e.g. Villa 1982; Lyman 1989; Morin et al., 2005).

\section{Conclusiones}

Este trabajo es un breve repaso a las publicaciones sobre remontajes óseos en zooarqueología, tafonomía y arqueología espacial. La incorporación de los remontajes como método en los estudios de los conjuntos faunísticos sigue siendo escasa en la actualidad. Desde su inicio en los años noventa del siglo pasado son pocos los zooarqueólogos que se han atrevido $-y$ atreven- a incluirlos en sus trabajos. A pesar de su relevante contribución su aplicación es limitada principalmente por la propia naturaleza de los huesos. Sus condiciones de conservación y preservación, a diferencia de la industria lítica, es uno de los principales factores que frena su desarrollo generalizado en los yacimientos. Esto, junto con el coste e inversión de tiempo necesario para su realización y la escasa generalización de su aplicación monitorizada o digitalizada, vienen a mostrarnos un estancamiento de las publicaciones, con sólo algunos ejemplos concretos y aislados recientes.

Este trabajo es una reflexión sobre la inclusión de los remontajes y su posible contribución en el análisis de los yacimientos arqueológicos. Los huesos remontados se convierten en ítems dinámicos que nos proporcionan información sobre los patrones de comportamiento de los grupos humanos en relación con el procesado, el desplazamiento de elementos o de partes esqueléticas, con la compartición de recursos cárnicos y/o el acondicionamiento del espacio, etc. Desde la tafonomía puede ayudarnos a descifrar palimpsestos, definir acciones, actividades y/o eventos de ocupación y, en definitiva, reconstruir procesos de formación del yacimiento. Y desde la arqueología espacial nos permiten inferir o interpretar por ejemplo conductas de organización social, económica, y/o espacial. 


\section{Bibliografía}

P. ANDREWS, J. COOK: "Natural modifications to bones in a temperate setting". MAN (N.S) 20 (1985): 675-691.

F. AUDOUZE, J.G. ENLOE: High resolution archaeology at Verberie: limits and interpretations. World Archaeology 29, 2 (1997), 195-207.

L. R. BINFORD: In pursuit of the Past. Decoding Archaeological Record. Thames and Hudson, London, 1983, 283.

L. R. BINFORD: "Etude taphonomique des restes fauniques de la Grotte Vaufrey. Couche VIII". In J.-P. Rigaud (Ed.): La Grotte Vaufrey a Cenac et Saint Julien (Dordogne): Paléoenvironnemente, chronologie et activités humaines. Societé Préhistorique Française, Paris, 1988, 535-564.

R. BLASCO, J. ROSELL, J. FERNÁNDEZ PERIS, I. CÁCERES, J.M. VERGÈS: New element of trampling: an experimental application on the Level XII faunal record of Bolomor Cave (Valencia, Spain). Journal of Archaeological Science 35(6) (2008), 1605-1618.

C.K. BRAIN: The hunters or the hunted? An introduction to African Cave Taphonomy. University Chicago Press, Chicago, 1981, 376.

P. BRAVO: Estudio zooarqueológico y de remontajes del subnivel Ja del Abric Romaní (Capellades, Barcelona): Tesis de licenciatura. Universitat Rovira i Virgili. Tarragona., 2001, 167.

E. CAMARÓS, M. CUETO, L.C. TEIRA, J. TAPIA, M. CUBAS, R. BLASCO, J. ROSELL, F. RIVALS: "Large carnivores as taphonomic agents of space modification: an experimental approach with archaeological implications". Journal of Archaeological Science 40, 2 (2013), 1361-1368.

M.G. CHACÓN, M.C. FERNÁNDEZ-LASO: "Modelos de ocupación durante el Paleolítico Medio: el nivel K del Abric Romaní (Capellades, Barcelona, España)": Complutum 18, (2007):47-60.

N.J. CONARD, T.J. PRINDIVILLE, D.S. ADLER: Reffiting bones and stones as a means of reconstruction middle Palolithic subsistence in the Rhineland. In J.P. BRUGAL, L. MEIGNEN, M. PATOU-MATHIS (Eds.): Économie préhistorique: les comportaments de subsistance au Paléolithique. Actes du XVIII Rencontres Internationales d'Archéologie et d'Histoire d'Antibes. Sophia Antipolis, Éds. APDCA, (1998), 273-290.

J. R. COOPER, F. QIU: "Expediting and standardizing stone artifact refitting using a computerized suitability model". Journal of Archaeological Science 33 (2006), 987-998.

H.T. BUNN et al: "Fxjj50: An Early Pleistocene site in northern Kenya". World Archaeology 12, 2 (1980), 109-136. 
E.S. CZIESLA, S. EICKHOFF, N. ARTS, y D. WINTER: The big puzzle: International Symposium on refitting stone artefacts. Holos Press, Bonn, 1990, 652.

J. G. ENLOE: Subsistence organization in the Upper Paleolithic: Carcass refitting and food sharing at Pincevent. Phd dissertation. Mexico, University of New Mexico, Alburquerque, 1991.

J.G. ENLOE: "Remontatge en zooarqueologia: tafonomía, economia i societat". Cota Zero 11 (1995): 31-37.

J.G. ENLOE, F. DAVID: Food sharing in the palaeolithic: carcass refitting at Pincevent. In J. L. HOFMAN, J. G. ENLOE (Eds.): Piecing Together the Past: Applications of refitting studies in archaeology. British Archaeological Reports International Series 578, Oxford, 1992, 296-299.

J.G. ENLOE, F. DAVID: "Le remontage des os par individus: le partage du renne chez les Magdaléniens de Pincevent (La Grande Paroisse, Seine-et-Marine)". Bulletin de la Société Prehistorique Française 86, 9 (1989), 275-281.

M.C. FERNÁNDEZ-LASO: Remontajes faunísticos y relaciones entre áreas domésticas en los niveles K, L y M del Abric Romaní (Capellades, Barcelona, España). Tesis Doctoral Inédita. Universitat Rovira i Virgili, Tarragona, 2010, 782.

M. J. GABUCIO: estudi espaciotemporal d'un palimpsest: anàlisi zooarqueològica i tafonòmica del nivell o de l'Abric Romaní (Capellades, Barcelona, Espanya). Tesis Doctoral Inédita. Universitat Rovira i Virgili, Tarragona, 2010, 331.

J. HALLOS: "15 minutes of Fame": Exploring the temporal dimension of middle Pleistocene lithic technology". Journal of Human Evolution 49 (2005), 15179.

A.S. HENSHAW: "Location and appropriation in the Artic: an integrative zooarchaeological approach to historic Inuit household economies". Journal of Anthropological Archaeology 18 (1999), 79-118.

J.L. HOFMAN: "The refitting of chipped-stone artifacts as analytical and interpretive tool". Current Anthropology 22 (1981), 691-693.

J.L. HOFMAN: "Vertical movement of artefacts in alluvial and stratified deposits". Current Anthropology 27, 2 (1986), 163-171.

J. L. HOFMAN: Putting the pieces together: Introduction to refitting. In J. L. HOFMAN y J. G. ENLOE: Piecing together the past: applications of refitting studies in archaeology. British Archaeological Reports International Series 578, Oxford, 1992a, 1-15.

J. L. HOFMAN: Defining Buried Occupation Surfaces in Terrace Sediments. In J.L. HOFMAN, J.G. ENLOE: Piecing Together the Past: Applications of Refitting Studies in Archaeology. British Archaeological Reports International Series 578, Oxford, 1992b, 128-150. 
J.L. HOFMAN, J. G. ENLOE (Eds.): Piecing together the past: applications of refitting studies in archaeology. British Archaeological Reports International Series 578, Oxford, 1992, 315.

E. JOHNSON: "Paleoindian bone expediency tools: Lubbock Lake and Bonefire Shelter". Canadian Journal of Anthropology 2 (1982), 145-157.

E. JOHNSON (Ed.): Cultural activities and interactions. In Lubbock Lake: Late Quaternary studies on the southern high plains. College Station. A \& M University Press, Texas, 1987, 120-158.

E.M. KROLL, G. ISAAC: Configurations of artifacts and bones at early Pleistocene sites in East Africa. In H.J. HIETALA: Intrasite Spatial Analysis in Archaeology. Cambridge University Press, Cambridge, 1984, 4-31.

M. L. LARSON, E.E. INGBAR: Perspectives on refitting: critique and complementary approach. In J. L. HOFMAN, J.G. ENLOE (Eds.): Piecing together the past: applications of refitting studies in archaeology. British Archaeological Reports International Series 578, Oxford 1992, 151-162.

A. LEROI-GOURHAN, M. BRÉZILLON: "L'habitation Magdaleniense No. 1 de Pincevent Pres Montereau (Seine-et-Marne)". Gallia Prehistorie 9 (1966), 263-371.

A. LEROI-GOURHAN, M. BRÉZILLON: "Fouilles de Pincevent. Essai d'analyse ethnographique d'un habitat magdalénien (la section 36)". Gallia Préhistoire VIle Supplément. 2 vols. 1972.

J. L. LOCHT: Modalités d'implantation et fonctionnement interne des sites. L'apport de trois gisements de plein air de la phase récente du Paléolithique moyen dans le nord de la France (Bettencourt-Saint-Ouen, Villiers-Adam et Beauvais). In N. J. CONARD (Ed.): Settlement Dynamics of the Middle Palaeolithic and Middle Stone Age, Kerns Verlag. Tübingen, 2001: 361-394.

L.R. LYMAN: Taphonomy of Cervids Killed by the May 18, 1980, Volcanic eruption of mount St. Helens, Washington, U.S.A In R. BONNICHSEN, M.H. Sorg (Eds.): Bone Modification. University of Maine Center for the Study of the First Americans. Orono, 1989, 149-167.

L.R. LYMAN: Vertebrate taphonomy. Cambridge University Press, Cambridge, 1994, 524.

L.R. LYMAN: "Identifying bilateral pairs of deer (Odocoileus sp.) bones: how symmetrical is symmetrical enough?". Journal of Archaeological Science 33 (2006), 1256-1265.

L.R. LYMAN: "(Zoo)Archaeological refitting a consideration of methods and analytical search radius. Journal of Anthropological Research 64 (2008), 229-248.

C.W. MAREAN, S.Y. KIM: "Mousterian large-mammal remains from Kobeh Cave. Behavioral implications for Neanderthals and early modern humans". Current Anthropology 39 (1998), 80-113. 
A. B. MARÍN ARROYO: Análisis arqueozoológico, tafonómico y de la distribución espacial de la fauna de mamíferos de la Cueva de la Fragua (Santoña, Cantabria). Ediciones TGD, Santander, 2004, 197.

A. B. MARÍN ARROYO: La fauna de mamíferos en el Cantábrico Oriental durante el Magdaleniense y Aziliense: nuevos enfoques y líneas de investigación arqueozoológicas. Tesis Doctoral Inédita. Universidad de Cantabria. Santander, $2007,332$.

E. MORIN, T. TSANOVA, N. SIRAKOV, W. RENDU, J.B. MALLYE, F. LÉVÊQUE: "Bone refits in stratified deposits: testing the chronological grain at SaintCésaire". Journal of Archaeological Science 32, 7 (2005), 1083-1098.

J. PARKINGTON, P. NILLSEN, C. REELER, C. HENSHILWOOD: "Making sense of space at Dunefield Midden Campsite, Western Cape, South Africa". Southern African Field Archaeology 1 (1992), 63-71.

D.J. RAPSON, L.C. TODD: Conjoins, Contemporaneity, and Site Structure: Distributional Analyses of the Bugas-Holding Site. In J.L. HOFMAN J.G. ENLOE: Piecing Together the Past: Applications of Refitting Studies in Archaeology. British Archaeological Reports International Series 578, Oxford, 1992, 238263.

W. ROEBROEKS: "From find scatters to early hominid behaviour. A study of middle Palaeolithic Riverside Settlments at Maastricht-Belvédère (the Netherlands)". Analecta Praehistorica Leidensia 21, 1988.

J. ROSELL, R. BLASCO, M.C. FERNÁNDEZ-LASO, M. VAQUERO, E. CARBONELL: Connecting areas: Faunal refits as a diagnostic element to identify syncronicity in the Abric Romaní archaeological assemblages. Quaternary International 225 (2012), 56-67.

M. B. SCHIFFER: "Toward the identification of formation processes". American Antiquity 48, 4 (1983), 675-706.

F.C.J. SPURRELL: "On the discovery of the place where Palaeolithic implements were made at Crayford". Quarterly Journal of the Geological Society 36 (1880), 544-549.

U.A. SCHURMANS: Refitting in the old and new worlds. In U. SCHURMANS y M. De BIE (Eds.): Fitting rocks: lithic refitting examined. British Archaeological Reports International Series 1596. Oxford, 2007, 7-23.

M. G. STEVENSON: Beyond the Formation of Hearth-Associated Artifact Assemblages. In E. M. Kroll, T. D. Price (Eds.): The Interpretation of Archaeological Spatial Patterning. New York, Plenum Press, 1991, 269-299.

L.C. TODD: Taphonomy of The Horner II bone bed. In G. C. FRISON, L.C. TODD (Eds.): The Horner Site: The type site of the cody cultural complex. Academy Press, Orlando, 1987, 107-198. 
L.C. TODD, D.J. STANFORD: Application of conjoined bone data to site structural studies. In J.L. HOFMAN, J.G. ENLOE (Eds.): Piecing together the past: applications of refitting studies in Archaeology. British Archaeological Reports International Series, 578. Oxford, 1992, 21-35.

L.C. TODD, G.C. FRISON: Reassembly of bison skeletons from The Horner Site: A study in anatomical refitting. In J.L. HOFMAN, J.G. ENLOE (Eds.): Putting the pieces together: An introduction to refitting. British Archaeological Reports International Series 578, Oxford, 1992, 63-82.

M. VAQUERO: "New perspectives on recycling of lithic resources using refitting and spatial data". Quartär 58 (2011), 113-130.

M. VAQUERO, M.G. CHACÓN, M.C. FERNÁNDEZ-LASO, K. MARTíNEZ, J.M. RANDO: Intrasite spatial patterning and transport in the Abric Romaní Middle Palaeolithic site (Capellades, Barcelona, Spain). In N.J. CONARD (Ed.): Settlement Dynamics of the Middle Palaeolithic and Middle Stone Age, Kerns Verlag. Tübingen, 2001, 573-595.

M. VAQUERO, A. BARGALLÓ, M.G. CHACÓN, F. ROMAGNOLI, P. SAÑUDO: "Lithic recycling in a Middle Paleolithic expedient context: Evidence from the Abric Romaní (Capellades, Spain)". Quaternary International, 361 (2015), 212228.

P. VILLA: "Conjoinable pieces and site formation processes". American Antiquity 47, 2 (1982), 276-290.

P. VILLA, C. BOUVILLE, J. COURTIN, D. HELMER, E. MAHIEU, P. SHIPMAN, G. BELLUOMINI, M. BRANCA: "Cannibalism in the Neolithic". Science 233 (1986), 431-437.

P. VILLA, E. MAHIEU: "Breakage patterns of human long bones". Journal of Human Evolution 21 (1991), 27-48.

M. VOORHIES: Taphomony adn population dynamics of an Early Pliocene vertebrate fauna, Knox County, Nebraska, University of Wyoming Contributions to Geology Special Paper 1, Laramie, 1969.

N. M. WAGUESPACK: "Caribou sharing and storage: refitting the Palangana site". Journal of Anthropological Archaeology 21 (2002), 396-417. 
